\title{
Modeling the Chromatic Correction Error in Adaptive Optics: Application to the Case of Mid-Infrared Observations in Dry to Wet Atmospheric Conditions
}

\author{
Laurent Jolissaint ${ }^{\mathrm{a}}$ and Sarah Kendrew \\ Leiden Observatory, University of Leiden
}

\begin{abstract}
In this paper we extend to the mid-IR previous work from other authors on the effect of the chromatic correction error in adaptive optics, including the contribution of atmospheric water vapor content. An analytic model of the closed-loop AO corrected residual phase spatial power spectrum is presented that includes the overall mixed effect of anisoplanatism, servo-lag and refractive index dispersion. Calculating the Strehl ratio loss as a function of the refractive index dispersion, we demonstrate that (1) the error variance grows with the optical turbulence phase variance, but thanks to the aberration-damping effect of the outer scale of optical turbulence for large apertures, chromatic correction error variance is much reduced in comparison with an infinite outer scale case and is not a significant issue for ELTs; (2) in the mid-IR wavelengths, where the fluctuations of the refractive index of air due to water absorption lines are particularly strong, chromatic correction error might become relatively significant in terms of wavefront error (in the range 10-100 nm), but is totally negligible when compared to the imaging wavelength, even in rather wet conditions (20 mm of total integrated column of water); (3) tight errors budget, with WFS in the VIS and correction in the NIR domain, is the only case where chromatic error might become an issue, as with extreme AO systems. The power spectrum model presented here can be used to evaluate the chromatic correction error and its contribution to the long exposure PSF, using the spatial frequency approach.
\end{abstract}

\section{Introduction}

The refractivity $N \equiv n-1$ of a gas is proportional to its density, and its variation with the wavelength follows lines profile laws (see [1] for an extensive description). Air refractivity is given by the sum of the refractivity of each of its constituents (see figure 1), and fluctuation of the air's density due to the turbulent mixing of air masses at different temperatures is at the origin of optical turbulence, for the correction of which adaptive optics systems (AO) have been devised. It is generally assumed that in the optical range $300 \mathrm{~nm}$ to $30 \mu \mathrm{m}$ - and outside of the regions of $\mathrm{H}_{2} \mathrm{O}$ and $\mathrm{CO}_{2}$ resonances, the dispersion of air's refractive index is very low, therefore optical turbulence can be considered in first order as being achromatic. Thanks to this, the optical bands of wavefront sensing and wavefront correction can be separated, allowing the use of any available photons from any guide star source, regardless of its color, to evaluate the wavefront aberration at the observation wavelength(s). In other words, this so-called chromatic correction error is negligible in classical AO systems.

Recently, this assumption has been re-examined (see for instance [2], [3], [4]). Indeed, it is expected that in the context of Extreme AO mode, particularly for large \& extremely large telescopes (large $D / r_{0}$ ratio), chromatic correction error is not necessarily a negligible component of the error budget anymore; therefore, it needs to be evaluated and techniques to compensate for it must be implemented, for instance as proposed in Ref. [2]. Beside, the impact of water vapor on the air refractive index became recently a (potential) concern for AO observations in the mid-IR region above $10 \mu \mathrm{m}$. Indeed, as can be seen in figure 1, refractivity departs significantly from the dry air value when water vapor content is increased from dry to mid-wet to wet conditions, and the question therefore remains wether this departure would generate a significant chromatic correction error for $\mathrm{AO}$ observation in the far end of the N-band and in the Q-band, assuming that wavefront sensing is done in the visible around $500 \mathrm{~nm}$.

\footnotetext{
a e-mail: jolissaint@strw.leidenuniv.nl
}

This is an Open Access article distributed under the terms of the Creative Commons Attribution-Noncommercial License, which permits unrestricted use, distribution, and reproduction in any noncommercial medium, provided the original work is properly cited. 
In order to study the chromatic correction error, Owner-Petersen [2] has initially developed a model of the spatial frequency power spectrum of the error, from which the phase error variance can be calculated. This model has shown that this error can become very significant in the case of large apertures, in the case of a fully developed Kolmogorov turbulence, i.e. with an infinite outer scale. Typical values of the outer scale, though, are in the range 10 to $100 \mathrm{~m}$ and this has a strong damping effect on the chromatic correction error. Owner-Petersen model is developed in the context of an open-loop model though, and makes use of a refractivity model (Ciddor [6]) that is not valid in the mid-IR range.

In the context of the study for a mid-IR AO instrument (METIS [7]), we wanted to have an accurate evaluation of the chromatic correction error and in particular, the impact of water vapor on AO performance. A closed-loop model of the chromatic correction error power spectrum was therefore needed, that included a generalist model of the air refractivity, for any wavelength. Quickly, it became evident that a model of the chromatic correction PSD could not be made independent of the servo-lag error PSD, and as anisoplanatism and servo-lag are not independent errors, we finally came up with what we call here the aniso-servo-dispersion error PSD, in closed loop. Alongside other closed loop errors (WFS aliasing and noise), this PSD was coded into our Fourier modeling code PAOLA, to produce the results presented in this paper.

Next section presents the refractivity model that we have been used. Then we give, without demonstration, the formulas for the closed loop aniso-servo-dispersion error PSD. Only results in terms of Strehl or wavefront error RMS are given here, i.e. we made only use of the integral of the PSD. The same PSD can be used otherwise to compute the associated long exposure PSF using the Fourier approach described in [8]. Applying our model on a median atmospheric case at Paranal Observatory, we analyze and discuss the effect of the telescope aperture diameter, the optical turbulence outer scale, and the amount of water vapor on $\mathrm{AO}$ performance in the near- and mid-IR domains.

\section{Earth's Atmosphere Refractive Index Model, from the Visible to the Mid-Infrared}

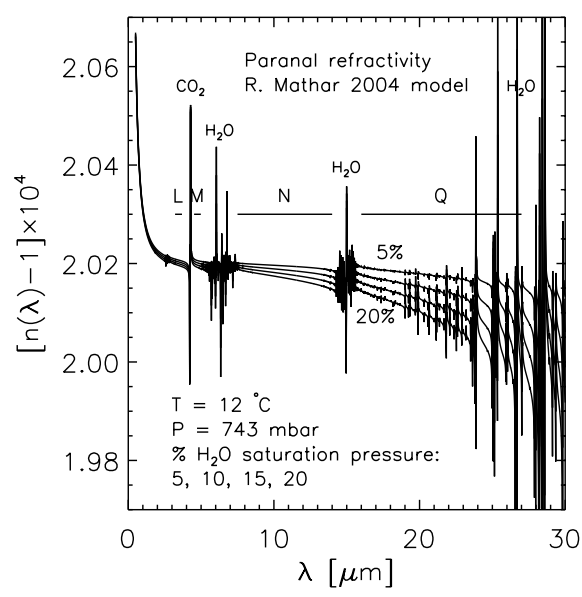

Fig. 1. Wet air refractivity over the whole optical range $300 \mathrm{~nm}$ to $30 \mathrm{mum}$, calculated using Mathar's on-line refractivity modeling tool.

$8 \mu \mathrm{m}$, nor the carbon dioxide or water vapor resonances. equation does
The most accurate model for the continuum of the air's refractivity in the visible and up to $1.7 \mu \mathrm{m}$ is given by Ciddor's empirical model [6], and is enough to evaluate the chromatic correction error in this range. The model can be extended without much consequences up to the end of K-band at $2.4 \mu \mathrm{m}$. Beyond this limit, $\mathrm{CO}_{2}$ and $\mathrm{H}_{2} \mathrm{O}$ resonances affect deeply the refractive index continuum, and Ciddor model should not be used anymore. For our study, we therefore use the work of Mathar [5] who computed accurately the moist air refractivity up to $30 \mu \mathrm{m}$, by adding the individual contributions of each transition line for water vapor and carbon dioxide, scaled with the HITRAN cross sections data base.

Figure 1 shows the Mathar's refractivity model used in our study, for Paranal observatory median temperature and pressure conditions, and four values of the partial saturation pressure that represent a dry to moderately wet atmosphere. Model was computed using Mathar's online tool ${ }^{1}$. One can see the strong impact of water vapor on the continuum after $8 \mu \mathrm{m}$, and the strong resonances in the Q-band. Ciddor equation does not reproduce the drop of the continuum after

\footnotetext{
${ }^{1}$ http://www.strw. leidenuniv.nl/ mathar/progs/prWaterWeb.html
} 


\section{Closed-loop residual wavefront spatial power spectrum model with refractive index dispersion}

The wavefront error after the optical beam has crossed a turbulent volume of thickness $\Delta h$ is given by the usual formula

$$
w_{\lambda}(\mathbf{r}, t)=\int_{0}^{\Delta h} N_{\lambda}(\mathbf{r}, h, t) \mathrm{d} h
$$

where $N_{\lambda}$ is the refractivity at wavelength $\lambda$. Now the question with chromatic correction error is: how can we determine the wavefront at the observation wavelength $\lambda_{\mathrm{DM}}$ knowing the wavefront at the sensing wavelength $\lambda_{\mathrm{wFS}}$ ? (Note that we assume narrow bands, to make the problem simpler). No need to invert the integral above to answer the question. Indeed, it is always possible to write back the refractivity as the refractivity for chosen normal pressure and temperature conditions times a geometrical function that will take care of the description of the local turbulent flow. The normal refractivity will be a function of the wavelength, the geometrical function will not. We assume therefore that we can write

$$
w_{\lambda}(\mathbf{r}, t)=N_{\lambda}^{\mathrm{n}} \int_{0}^{\Delta h} \xi(\mathbf{r}, h, t) \mathrm{d} h \quad \text { i.e. } \quad w_{\lambda} \sim N_{\lambda}^{\mathrm{n}}
$$

where $\xi$ is achromatic, and has a structure that has no importance for us here. The only thing which counts is that the wavefront at a given point in the pupil $\mathbf{r}$ and at a given instant is assumed to be proportional to the refractivity at some normal conditions, the choice of which is free. Therefore, we can compute the wavefront error at the observation (or correction) wavelength from the wavefront measure using

$$
w_{\lambda_{\mathrm{DM}}}=\frac{N_{\lambda_{\mathrm{DM}}}^{n}}{N_{\lambda_{\mathrm{WFS}}}^{n}} w_{\lambda_{\mathrm{WFS}}} \equiv v\left(\lambda_{\mathrm{DM}}, \lambda_{\mathrm{WFS}}\right) w_{\lambda_{\mathrm{WFS}}}
$$

where we define $v\left(\lambda_{\mathrm{DM}}, \lambda_{\mathrm{WFS}}\right)$, the refractivity ratio, as the dispersion factor.

Let us introduce now the fundamental equation of $\mathrm{AO}$, separating the sensing and correction wavelength as we did above. The wavelength of interest for the residual wavefront error $w[r]$ is the correction (science channel) wavelength, but the wavelength associated to the measure, or the applied correction $w[c]$, is the WFS wavelength, so we have

$$
w[r]_{\lambda_{\mathrm{DM}}}(\mathbf{r}, \boldsymbol{\theta}, t)=w[a]_{\lambda_{\mathrm{DM}}}(\mathbf{r}, \boldsymbol{\theta}, t)-w[c]_{\lambda_{\mathrm{WFS}}}(\mathbf{r}, t)
$$

where $w[a]_{\lambda_{\mathrm{DM}}}$ indicates the input atmospheric turbulent wavefront error, and $\boldsymbol{\theta}$ is the angular separation between the science object and the guide star. Following the same approach than the one described in our initial paper on the spatial frequency method [8], but in a closed loop scheme, and including the dispersion factor $v\left(\lambda_{\mathrm{DM}}, \lambda_{\mathrm{WFS}}\right)$, we have computed the four components of the residual wavefront spatial power spectrum (given without demonstration here):

$$
\Xi\left\{w[r]_{\lambda_{\mathrm{DM}}}\right\}(\mathbf{f})=\Xi\left\{w[\mathrm{HF}]_{\lambda_{\mathrm{DM}}}\right\}(\mathbf{f})+\Xi\left\{w[\mathrm{AS}]_{\lambda_{\mathrm{DM}}}\right\}(\mathbf{f})+\Xi\left\{w[\mathrm{AL}]_{\lambda_{\mathrm{DM}}}\right\}(\mathbf{f})+\Xi\left\{w[\mathrm{NS}]_{\lambda_{\mathrm{DM}}}\right\}(\mathbf{f})
$$

First term is the wavefront high frequency error, i.e. aberrations at spatial frequencies above the system cut-off spatial frequency $f_{\mathrm{AO}}=1 /(2$ pitch $)$, that cannot be seen then cannot be corrected by the system. Using the Von Karmán spatial power spectrum of the phase, we get (as in open loop)

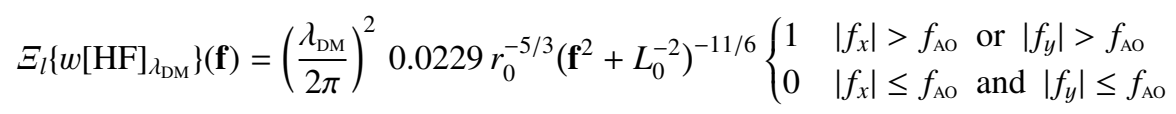

Second term is the aniso-servo-dispersion error and is the one we are interested in. As are the two next terms, this error is at low spatial frequency: it involves aberrations at frequencies that are seen by the 
system, but are only partly corrected, because of anisoplanatism, servo-lag, and chromatic correction. We find that this error can be written as the atmospheric turbulent wavefront spatial power spectrum filtered by the so-called aniso-servo-dispersion transfer function $\mathcal{T}_{\text {asd }}$ (a low pass function). For the turbulent layer $l$ at altitude $h_{l}$ and wind velocity $\mathbf{v}_{l}$, the aniso-servo-dispersion PSD writes

$$
\Xi_{l}\left\{w[\mathrm{AS}]_{\lambda_{\mathrm{DM}}}\right\}(\mathbf{f})=\left(\frac{\lambda_{\mathrm{DM}}}{2 \pi}\right)^{2} 0.0229 r_{0, l}^{-5 / 3}\left(\mathbf{f}^{2}+L_{0}^{-2}\right)^{-11 / 6} \mathcal{T}_{\text {asd }, l}(\mathbf{f}) \begin{cases}0 & \left|f_{x}\right|>f_{\mathrm{AO}} \text { or }\left|f_{y}\right|>f_{\mathrm{AO}} \\ 1 & \left|f_{x}\right| \leq f_{\mathrm{AO}} \text { and }\left|f_{y}\right| \leq f_{\mathrm{AO}}\end{cases}
$$

with

$$
\mathcal{T}_{\text {asd }, l}(\mathbf{f})=\frac{\text { num }}{\operatorname{den}}
$$

where

$$
\begin{aligned}
\text { num } & =1-\cos \left(2 \pi \Delta t \mathbf{f} \cdot \mathbf{v}_{l}\right) \\
& +g \Gamma_{\mathrm{DM}}(\mathbf{f}) \operatorname{sinc}\left(\Delta t \mathbf{f} \cdot \mathbf{v}_{l}\right)\left\{\cos \left[2 \pi(\Delta t / 2+\tau) \mathbf{f} \cdot \mathbf{v}_{l}\right]-\cos \left[2 \pi(\Delta t / 2-\tau) \mathbf{f} \cdot \mathbf{v}_{l}\right]\right\} \\
& -v^{-1}\left(\lambda_{\mathrm{DM}}, \lambda_{\mathrm{WFS}}\right) g \Gamma_{\mathrm{DM}}^{2}(\mathbf{f}) \operatorname{sinc}\left(\Delta t \mathbf{f} \cdot \mathbf{v}_{l}\right) \times \\
& \left\{\cos \left[2 \pi(\Delta t / 2+\tau) \mathbf{f} \cdot \mathbf{v}_{l}-2 \pi h_{l} \mathbf{f} \cdot \boldsymbol{\theta}\right]-\cos \left[2 \pi(\Delta t / 2-\tau) \mathbf{f} \cdot \mathbf{v}_{l}+2 \pi h_{l} \mathbf{f} \cdot \boldsymbol{\theta}\right]\right\} \\
& -v^{-1}\left(\lambda_{\mathrm{DM}}, \lambda_{\mathrm{WFS}}\right) g^{2} \Gamma_{\mathrm{DM}}^{3}(\mathbf{f}) \operatorname{sinc}^{2}\left(\Delta t \mathbf{f} \cdot \mathbf{v}_{l}\right) \cos \left(2 \pi h_{l} \mathbf{f} \cdot \boldsymbol{\theta}\right) \\
& +1 / 2 g^{2} \Gamma_{\mathrm{DM}}^{2}(\mathbf{f}) \operatorname{sinc}^{2}\left(\Delta t \mathbf{f} \cdot \mathbf{v}_{l}\right)\left[1+v^{-2}\left(\lambda_{\mathrm{DM}}, \lambda_{\mathrm{WFS}}\right) \Gamma_{\mathrm{DM}}^{2}(\mathbf{f})\right]
\end{aligned}
$$

and

$$
\begin{aligned}
\text { den } & =1-\cos \left(2 \pi \Delta t \mathbf{f} \cdot \mathbf{v}_{l}\right) \\
& +g \Gamma_{\mathrm{DM}}(\mathbf{f}) \operatorname{sinc}\left(\Delta t \mathbf{f} \cdot \mathbf{v}_{l}\right)\left\{\cos \left[2 \pi(\Delta t / 2+\tau) \mathbf{f} \cdot \mathbf{v}_{l}\right]-\cos \left[2 \pi(\Delta t / 2-\tau) \mathbf{f} \cdot \mathbf{v}_{l}\right]\right\} \\
& +1 / 2 g^{2} \Gamma_{\mathrm{DM}}^{2}(\mathbf{f}) \operatorname{sinc}^{2}\left(\Delta t \mathbf{f} \cdot \mathbf{v}_{l}\right)
\end{aligned}
$$

The total aniso-servo-dispersion PSD is simply given by the sum of each layer's PSD (we assume $N_{l}$ independent layers)

$$
\Xi\left\{w[\mathrm{HF}]_{\lambda_{\mathrm{DM}}}\right\}(\mathbf{f})=\sum_{l=1}^{N_{l}} \Xi_{l}\left\{w[\mathrm{HF}]_{\lambda_{\mathrm{DM}}}\right\}(\mathbf{f})
$$

and the different parameters appearing in the equations are given in table 1.

Table 1. Parameters of the aniso-servo-dispersion PSD

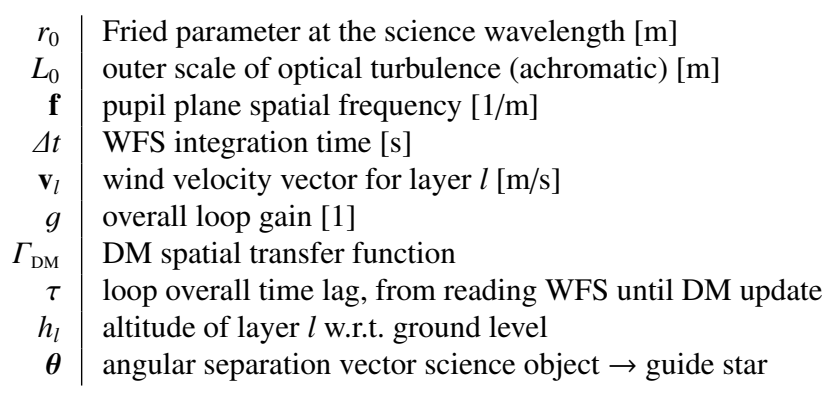

The WFS spatial aliasing and WFS noise PSD are not given here. These terms do not depend on the dispersion. The full closed loop spatial frequency model will be described in a forthcoming publication. 


\section{Analysis of chromatic correction error for moderate and large apertures}

We now evaluate the impact of chromatic correction. We will chose a test case that represents the parameter space for METIS a mid-IR AO instrument studied for the European ELT. Results are also given for a $6.5 \mathrm{~m}$ aperture, to serve as a comparison with moderate scale telescopes. Parameters of the modeling are given in table 2 .

\begin{tabular}{r|l} 
Guide star & $\mathrm{mV} \mathrm{12,} \mathrm{G2-like} \mathrm{spectrum}$ \\
Zenith distance & $30^{\circ}$ \\
Seeing angle & $0.65 \%$ at zenith \\
Outer scale & $27 \mathrm{~m}, 100 \mathrm{~m}$, infinite \\
$C_{N}^{2}$ and wind profiles & median at Paranal Obs. \\
wind speed & $16.5 \mathrm{~m} / \mathrm{s}$ \\
Telescope diameter & $6.5 \mathrm{and} 42 \mathrm{~m}$, monolithic \\
DM and WFS pitch & $50 \mathrm{~cm}$ \\
WFS sensing wavelength & $0.5 \mu \mathrm{m}$ \\
Science wavelengths & 0.5 to $30 \mu \mathrm{m}$ \\
WFS integration time & optimized, 1.43 ms \\
Loop gain & optimized, 0.41 \\
Loop lag & $2 \mathrm{~ms}$ \\
WFS detector RON & $3 \mathrm{e} / \mathrm{px}$ \\
WFS FoV & $6-$ by-6 pixels Nyquist ltd.
\end{tabular}

Table 2. Parameters for the PAOLA-based model.
As we have seen, there is no such thing as a chromatic correction error that can be separated from the aniso-servo error, and for this reason, we have given the full equation. Now, this does not prevent us, once the anisoservo-dispersion error variance is calculated, to subtract the aniso-servo variance just to see how much of the error comes from dispersion. This is what we did and what we are showing in figure 2. On the left figure is shown the chromatic correction wavefront error, obtained from the chromatic correction variance.

On the amplitude of the error, and the effect of water vapor The error goes up quickly when the observing wavelength increases above the sensing wavelength, but rapidly reaches a plateau near $3 \mu \mathrm{m}$; up to

about $15 \mu \mathrm{m}$, the error, in terms of wavefront error, not phase, is basically stationary, therefore we can say that the mid-IR regime is certainly not more affected by the chromatic correction than the near-IR regime. For longer wavelength, though, water vapor has a strong influence on the wavefront error, but if we report it to the wavelength, the actual impact on performance is totally negligible. This can be seen on the right figure, where the associated Strehl is shown. Fluctuations due to water vapor above $20 \mu \mathrm{m}$ simply cannot be seen on the Strehl.
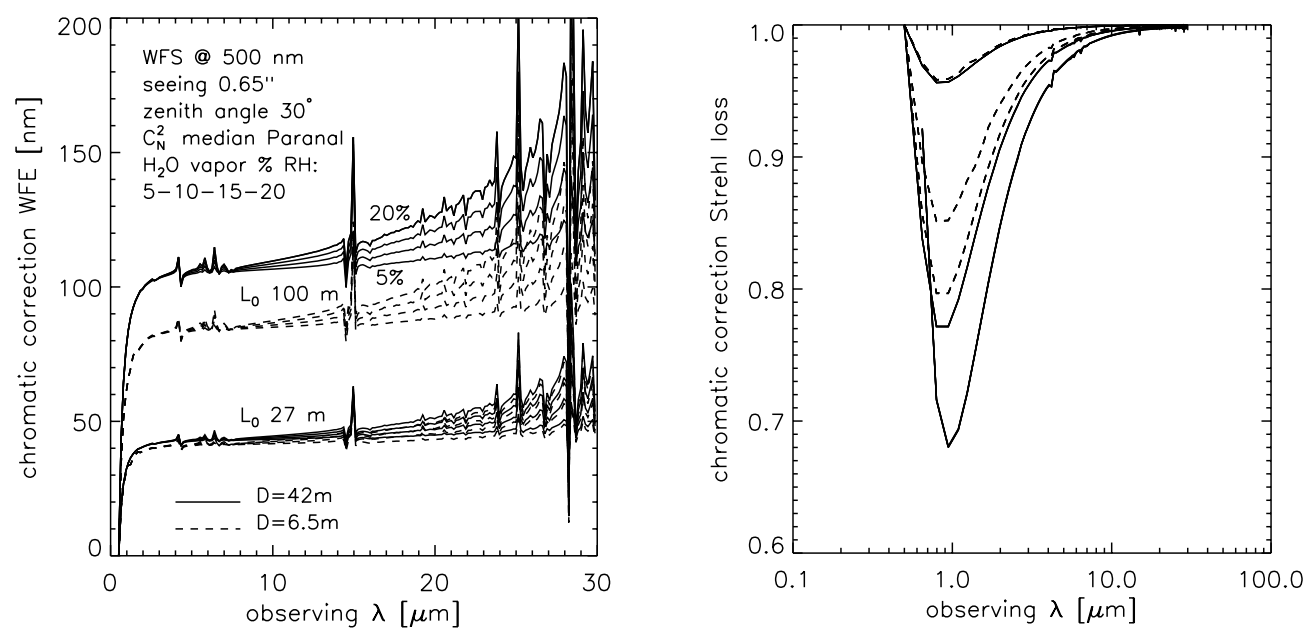

Fig. 2. Chromatic correction error. Left: wavefront error RMS. Right: associated Strehl loss. Conditions of the calculation are indicated. Continuous curves: telescope diameter $42 \mathrm{~m}$, dashed curves, $6.5 \mathrm{~m}$. On the Strehl figure, bottom curves are for an infinite outer scale, middle for $L_{0}=100 \mathrm{~m}$, and top for $L_{0}=27 \mathrm{~m}$. Wavefront sensing wavelength is $500 \mathrm{~nm}$ for both figures. 
On the effect of outer scale Let us consider the Strehl figure. It shows several curves for several values of the outer scale and the telescope diameter. From the lower to the upper curves, we have $L_{0}$ infinite, equal to $100 \mathrm{~m}$, and $27 \mathrm{~m}$. We see that if the outer scale was infinite, then the effect could be rather severe for near-IR wavelengths. In real life, fortunately, this never happens, and outer scales larger than $100 \mathrm{~m}$ occurs less than $3 \%$ of the time at Paranal [9]. According to the same source, the median value is around $20 \mathrm{~m}$, so we are even a bit conservative with the $27 \mathrm{~m}$ value. In any case, what we can see is that for the later value of $L_{0}$, the maximum impact on the Strehl is a drop of $4 \%$ only, around $1 \mu \mathrm{m}$. And the Strehl loss is less than $1 \%$ above $3 \mu \mathrm{m}$.

On the aperture size Large apertures are more affected by chromatic correction than smaller ones, but again the outer scale is washing away the differences. As we can see, for the value $L_{0}$ at $27 \mathrm{~m}$, the impact of the error is the same for a relatively small $(6.5 \mathrm{~m})$ than for the large aperture of $42 \mathrm{~m}$. This is easily explained by the fact that the aniso-servo-dispersion error variance is proportional to the turbulent phase variance itself. So again, thanks to the damping effect of outer scale, we can say that chromatic correction is no more a concern for ELTs than for smaller, more classical apertures.

On extreme AO systems We have seen in the Strehl figure that chromatic correction has an impact mostly in the near-IR region. Relatively independently from the aperture diameter, we can say that the WFE is about $40 \mathrm{~nm}$. It is probable that for Extreme AO (EXAO) systems working in the NIR regime, this WFE cannot be tolerated. Compensation scheme would be needed, either based on a model of the dispersion factor, or a try-and-error algorithms.

\section{Conclusions}

A model of the chromatic correction error is developed in this paper. It is demonstrated that this error affects only the visible to near-infrared part of the spectrum. In principle the error would be large for a ELT, but thanks to the damping effect of the outer scale of turbulence, this error is reduced to a rather modest level. As a rule of thumb, for classical seeing conditions, an OPD error of about $40 \mathrm{~nm}$ can be attributed to this error, independently of the telescope diameter, and the wavelength. Regarding the effect of water vapor, we find that even if the impact in terms of OPD error is significant above $15 \mu \mathrm{m}$, when reported to the wavelength, it becomes totally negligible. To conclude, water vapor is not an issue, and the only domain where chromatic correction do matter is extreme AO in the near-IR.

\section{References}

1. R. J. Hill et al., JOSA 70, (1980) 1192-1205

2. M. Owner-Petersen \& A. Goncharov, SPIE 5489, (2004) 507-517

3. M. Owner-Petersen, SPIE 6272, (2006) 62722F

4. N. Devaney et al., Applied Optics 47, (2008) 1072-1081

5. R. Mathar, Applied Optics 43, (2004) 928-932

6. P. Ciddor, Applied Optics 35, (1996) 1566

7. B. Brandl et al., SPIE 7015, (2008)

8. L. Jolissaint et al., JOSA A 23, (2006) 382-394

9. R. Conan et al., SPIE 4006, (2000) 963-973 